\title{
Editorial
}

\section{El ejercicio médico en el mundo: de la legislación a la práctica}

\author{
Jorge Iván Parra-Ardila*
}

Daniel Felipe Vera-Osorio*

*Estudiante de Medicina de V año, Facultad de Salud, Universidad Industrial de Santander

Correspondencia: Jorge Ivan Parra Ardila. Dirección: Calle 103c \# 13e13 Jardines de Coaviconsa, Bucaramanga, Colombia. Teléfono: 3134091756. Correo electrónico: jorgeivanpa-17@hotmail.com.

¿Cómo citar este artículo?: Parra-Ardila JI, Vera-Osorio DF. El ejercicio médico en el mundo: De la legislación a la práctica. MÉD. UIS.2019;32(3):9-12. doi:10.18273/revmed.v32n2-2019001

La violencia contra los médicos es un fenómeno que se ha desarrollado de manera progresiva con el paso de los años. Esta problemática ha desencadenado una serie de escenarios en los cuales los pacientes se han familiarizado con la idea de tener un trato inapropiado con los profesionales en este campo al momento de llevar a cabo su trabajo. Debido a esto, se cuestiona si el sistema sanitario, diseñado para asegurar que todas las personas cuenten con una calidad de vida óptima, debería tener ciertos parámetros que se basen tanto en quienes requieren de estos servicios como aquellos que los prestan, para que las relaciones médico paciente se fundamenten en el respeto y, que, de no ser así, exista una protección efectiva a los derechos que sean violados.

Una de las labores más importantes de un médico es generar empatía y confianza en su paciente para poder brindarle un servicio de mayor calidad que le permita cumplir con el objetivo de su trabajo de manera exitosa, sin embargo, esto no puede lograrse sin la disposición de sus pacientes. De las agresiones laborales, la Organización Internacional de Trabajo señala que el $25 \%$ son contra el personal sanitario. Debido a esto, algunas instituciones de salud se han enfocado en el estudio de la problemática y su afectación directa sobre el recurso humano1. Por ende, se han buscado alternativas que permitan la mejoría del sistema, criticado y polemizado, mediante la implementación de pautas que protejan a los médicos en el momento que se encuentren en una situación de vulnerabilidad.

\section{Ámbito internacional (situación global)}

Históricamente, la aparición de la misión médica surgió en el siglo XIX con el objetivo de ayudar a los combatientes heridos en los enfrentamientos sobre el escenario de la batalla de Solferino ${ }^{2}$. Este objetivo se encuentra plasmado en el Convenio de Ginebra, y actualmente son labores realizadas por la Cruz Roja, una entidad de carácter humanitario que se encuentra ligada al Derecho Internacional Humanitario (DIH), asegurando la protección de las personas afectadas por contingencias ocasionales, con absoluta imparcialidad, sin distinción alguna de sexo, raza, religión u otra característica. Además, otorga una connotación especial a los hospitales, demostrando que estos lugares no podían ser atacados arbitrariamente, y que se esperaba que fueran respetados por las partes involucradas en el conflicto ${ }^{3}$.

En la actualidad, la Asociación Médica Mundial ha mostrado una profunda preocupación por el crecimiento de los casos de agresión, tanto verbal como física, hacia el personal médico. En su reciente reunión con el Consejo de Oslo, más de 40 delegados alrededor del mundo expusieron las diferentes situaciones que se han presentado en sus países respectivos que demuestran el aumento de esta problemática. "Todos tienen el derecho a trabajar en un entorno seguro. La violencia contra los médicos es particularmente irracional, ya que tiene un impacto en todo el sistema de salud, incluida la atención de pacientes", expresó el Dr. Xavier Deau, 
presidente de la Asociación Médica Mundial4. El hecho de que la violencia contra los médicos sea un fenómeno presente internacionalmente hizo que algunos organismos, tales como la Organización Internacional del Trabajo, la Organización Mundial de la Salud, el Consejo Internacional de Enfermeras y la Internacional de Servicios Públicos se pronunciaran frente a esto, elaborando lo que hoy en día se conoce como las "Directrices marco para afrontar la Violencia Laboral en el Sector de la Salud" que tienen como objetivo servir de orientación general para abordar la violencia en el trabajo en el sector de salud ${ }^{5}$.

Esta situación se agrava cuando es normalizada por los pacientes, ya que muchas veces se promueve la idea de que los médicos son los culpables de los malos tratos a los que son sometidos, ya que no cumplen con su trabajo de manera eficaz. Michelle Mahon $^{6}$, representante del sindicato National Nurses United, se pronunció frente a este tema diciendo que en variadas ocasiones cuando alguien que hace parte del personal en el campo de la salud alza su voz frente a un episodio en el que haya tenido que vivir una situación violenta, "se los trata como si fueran ellos o ellas los que no saben cómo hacer su trabajo. Se los culpa de que haya sucedido".

Distintos países han buscado obtener soluciones por medio de la legislación para poder tener un respaldo frente a un hecho generador que pueda desencadenar una conducta punible, incluso se ha optado por proponer una reforma penal en la cual se tenga en cuenta este tipo de agresiones y quienes las sufren se vean amparados por la ley. Tal es el caso de la legislación española en la cual mediante trabajos investigativos se ha planteado la opción de una reforma legislativa, donde la labor desempeñada por el personal de salud deba tener una protección penal específica, ya que su ejercicio sobrepasa los intereses personales; por tanto, esta reforma "deberá seguir respaldada por los colegios de médicos y enfermeros como encargados de promocionar su profesionalismo, la normativa y planes de prevención de riesgos; y complementada por, tal vez, un régimen de infracciones y sanciones que diseñen las administraciones sanitarias $y$, por supuesto, por una reforma procesal"'. En un estudio realizado por Travetto $C$ en la cual por medio de una encuesta electrónica a través del sitio web IntraMed a más de 19000 profesionales en el campo de la salud, entre el 3 de junio y el 17 de diciembre de 2013, concluyendo que la manera más idónea para alcanzar un cambio positivo frente a esta problemática era el uso de herramientas complementarias que podrían ayudar a mitigar la violencia contra los médicos como la presencia policial o la seguridad privada, y otros derivados de estos, los cuales son: "la atención grupal, cámaras de seguridad, dispositivos antipánico, atención con puertas abiertas o prohibición del ingreso de familiares de los pacientes"8.

\section{Ámbito nacional (situación local)}

En Colombia, la situación en la que se encuentran los médicos siempre ha sido una cuestión en la cual muchos derechos se ven vulnerados. Con el comienzo del conflicto armado caracterizado por ser una guerra entre el Estado, grupos guerrilleros y paramilitares se acentuó la violencia hacia el personal médico, ya que los grupos subversivos centraron sus ataques en ciertas poblaciones donde ejercía el personal. El año 2002 fue un período en el cual las cifras de casos de agresiones que se presentaron hacia los doctores tuvo un auge notable, puesto que se rompió el proceso de paz con el grupo guerrillero FARC, iniciado durante el gobierno de Andrés Pastrana, lo que llevó a que este grupo intensificara sus acciones contra distintos sectores. Una investigación realizada por el Observatorio del Programa Presidencial de Derechos Humanos y DIH expone que es "evidente el incremento de las infracciones en el 2002, sobre todo las relacionadas con ataques a transportes sanitarios con 16 registros y lesiones en persona protegida con doce infracciones y 16 víctimas" ${ }^{\prime \prime}$.

Ya que esta problemática estaba creciendo de manera desmedida, el gobierno decidió tomar cartas en el asunto y de manera paulatina, ha planteado diferentes leyes para que estas personas cuenten con una protección por parte de la legislatura colombiana y así poder hacer efectivos sus derechos en el momento en el que se presente una posible violación de los mismos. Un ejemplo de esto es la Resolución No 01020 de 2002 expedida por el MINISTERIO DE SALUD de Colombia, mediante la cual se dictan medidas para la protección de la Misión Médica que, entre otras cosas, tiene como objeto principal los siguientes puntos:

1. "Fortalecer las garantías para brindar asistencia y protección humanitaria a la población más vulnerable afectada como consecuencia de conflictos armados, desastres naturales y otras calamidades.

2. Proteger, garantizar y facilitar la misión del 


\section{Mayo - Agosto}

personal médico, paramédico y sanitario, civil y religioso, que presta sus servicios para aliviar la suerte de las víctimas de los conflictos armados, desastres naturales y otras calamidades.

3. Proteger y garantizar los servicios que prestan las unidades sanitarias y los medios de transporte sanitarios públicos o privados" (Resolución No 01020 de 2002).

Otras medidas que se han tomado para regular la misión médica han sido el Decreto 860 de 1998, la Ley 852 de 2003, la Ley 875 de 2004, el Decreto 138 de 2005, el Decreto 2816 de 2006 y la Resolución 4481 de 2012. Cuando una agresión es causada a una persona que hace parte de la misión médica, este acto está en contra de lo pactado en el Convenio de Ginebra, y por ende significa una violación directa a los derechos humanos, por lo que el Estado colombiano ha promulgado las normativas anteriormente señaladas para cumplir con la efectividad y protección del bloque de constitucionalidad. Este último plantea que la legislación interna de nuestro país debe ser acorde a lo pactado en los tratados internacionales y armonizarse con el DIH y que estas normas y principios serán utilizados como "parámetros del control de constitucionalidad de las leyes, por cuanto han sido normativamente integrados a la Constitución, por diversas vías y por mandato de la propia Constitución" (Sentencia C-067/2003, CORTE CONSTITUCIONAL Colombiana).

Sin embargo, estos casos de violaciones a los derechos humanos siguen presentándose con frecuencia, así se ve plasmado en una encuesta realizada recientemente por la Asociación Colombiana de Hospitales y Clínicas aplicada a 630 trabajadores de la salud, en la que se encontró que el 98,7 por ciento de los funcionarios han sido víctimas de violencia verbal y el 27 por ciento de violencia física ${ }^{10}$. Lo anterior permite evidenciar que aunque existe una legislación que busca proteger los derechos de los médicos, esto no es suficiente para que aquellas situaciones cesen. Debido a esto se ha intentado implementar otras alternativas como una educación integral en la cual se enseñe a las personas a tratar a los profesionales con respeto y mesura, comprendiendo que la labor de un médico requiere de mucha empatía para que ambas partes, tanto el paciente como el trabajador se vean beneficiados.

\section{Conclusión}

La violencia contra los médicos es un fenómeno que afecta mundialmente y con frecuencia. Sin embargo, el DIH se ha encargado de proteger y amparar a aquellas instituciones como la Cruz Roja, que se encargan de hacer que la labor de estos trabajadores sea más amena y humana, cumpliendo con el fin de servir a la comunidad y salvar vidas. Además de esto, también se han creado acuerdos y tratados en los cuales se ratifica la importancia de los médicos y el personal sanitario en general; uno de estos es el capítulo II del Convenio de Ginebra, que les da una connotación especial a los hospitales, clínicas y distintos recintos, en los cuales se preste el servicio de la salud.

En Colombia, a causa del conflicto armado presente hace más de 50 años, esta situación se agravó, y aunque el gobierno ha tenido la intención de mitigarlo, logrando disminuir el impacto de la violencia en este ámbito, aún se siguen presentando casos de agresión física y verbal. La legislación colombiana también ha propuesto diferentes pautas, en las cuales se respaldan los trabajadores que pasan por una de estas situaciones y deciden tomar medidas para que se reparen los daños causados.

Además de esto, se deben implementar otras herramientas para que las personas que requieren los servicios de salud, ya sea pública o privada, tengan un conocimiento de las cifras que se ven reflejadas en distintos estudios realizados a lo largo de los años, en los cuales se demuestra que los médicos se encuentran en una situación de vulnerabilidad al tener que convivir con pacientes que no saben tener un trato adecuado con ellos y que de esta manera, se genere conciencia sobre el impacto negativo que una actitud errónea puede generar en el trabajo de quienes se encuentran en este campo. Una manera de lograr esto es por medio de campañas informativas en las cuales se muestren los índices de violencia contra los médicos presentes en la actualidad, las acciones legales que se pueden tomar frente a alguna situación de esta magnitud y, sobre todo, enfocar esto a crear una sociedad más educada y respetuosa frente a las personas que prestan un servicio, sea en el campo de la salud o cualquier otro en el que se presente esta problemática. 


\section{Referencias bibliográficas}

1. Ancalli-Calizaya, F., Cohaila, G., \& Maquera-Afaray, J. Agresiones contra el trabajador de salud en Tacna, Perú. Rev Peru Med Exp Salud Pública. 2014; 29(3)

2. Pablo Saz Peiró. Agresiones a sanitarios: "El médico a palos". Medicina naturista. 2018; 12 (1)

3. ¿Qué es el derecho internacional humanitario? Servicio de asesoramiento en derecho internacional humanitario. 2004.

4. Cuando la relación médico-paciente se distorsiona. Consenso Salud. [Internet].2015 [citado 11 Aug 2019]. (47): 4-7. Recuperado a partir de: http://periodico.consensosalud.com.ar/ consenso_n47.html.

5. Organización Internacional del Trabajo/Consejo Internacional de Enfermeras/ Organización Mundial de la Salud/Internacional de Servicios Públicos Directrices marco para afrontar la violencia laboral en el sector de la salud. Ginebra, Organización Internacional del Trabajo, 2002.

6. Harris-Taylor, M. ¿Por qué crece la violencia contra médicos
MÉD.UIS. 2019;32(2):9-12

y enfermeras? [Internet]. El Tiempo Latino. 10 de junio de 2019. Recuperado a partir de: http://eltiempolatino. com/news/2019/jun/10/por-que-crece-la-violencia-contramedicos-y-enferm/

7. Elena Martínez-Zaporta Aréchaga, Rafael Fernández-Delgado Momparler. Las agresiones a personal sanitario: propuesta para una reforma penal. DS. 2008; 16, (2)

8. Travetto C, Daciuk N, Fernández S, Ortiz P, Mastandueno R, Prats $\mathrm{M}$, et al. Revista panamericana de salud publica. [Internet]. Vol. 38, Rev. Panam. Salud Públ.; 2015 [citado 2019 Aug 10]. 307-315 p. Recuperado a partir de: https://scielosp.org/pdf/rpsp/2015. v38n4/307-315/es

9. Observatorio del programa presidencial para los derechos humanos y el DIH [Internet]. Bogotá D.C; 2005 [citado 2019 Aug 11]. Recuperado a partir de: http://www.derechoshumanos.gov. co/observatorio

10. Asociacion Colombiana de Hospitales y Clinicas - ACHC. Encuesta de las agresiones en el servicio de urgencias de las clínicas y hospitales afiliados a la ACHC. Revista Hospitalaria. 2015;103(septiembre-octubre de 2015):4-24. 